\title{
Distribution of Suitable Environments for Phlebotomus chinensis as the Vector for Mountain-Type Zoonotic Visceral Leishmaniasis - Six Provinces, China
}

\author{
Xinyi Wang ${ }^{1,2}$; Jingbo Xue ${ }^{1,2}$; Shang Xia ${ }^{1,2}$; Shuai Han ${ }^{1,2}$; Xiaokang $\mathrm{Hu}^{1,2}$; \\ Zhengbin Zhou ${ }^{1,2}$; Yi Zhang ${ }^{1,2}$; Shizhu Li ${ }^{1,2, \#}$
}

\section{Summary \\ What is already known on this topic? \\ Phlebotomus chinensis ( $P$. chinensis) is a sandfly and the main vector of mountain-type zoonotic visceral leishmaniasis (MT-ZVL) in China. However, the distribution of suitable environments for the vector has not been studied yet. \\ What is added by this report? \\ This study found that temperate hilly zones in midwestern China are suitable for $P$. chinensis survival with appropriate environmental factors such as moderate normalized difference vegetation value (NDVI), land use type, landform, temperature, and vegetation. Suitable living conditions for the high- density $P$. chinensis that caused the reemergence of MT-ZVL already existed. \\ What are the implications for public health practice? \\ Targeted strategies should be implemented to control the vector and the reemergence of MT-ZVL, such as by strengthening key environment monitoring and taking accurate measures for residents, mobile and migrant populations.}

Phlebotomus chinensis ( $P$. chinensis) is a sandfly that is the main vector of mountain-type zoonotic visceral leishmaniasis (MT-ZVL) in China, an endemic infectious disease caused by Leishmania spp. parasitizing in the human lymphocyte system (1-2). To analyze the distribution of suitable environments for the $P$. chinensis vector and control the reemergence of MT-ZVL, the distribution data of $P$. chinensis that were previously documented and a national survey in 2016 were input into MaxEnt software to calculate the ecological suitability results. The impact of key environmental factors on the vector were determined, and suitable living conditions for high-density $P$. chinensis that caused the reemergence of MT-ZVL already existed. Strengthening key environment monitoring and taking accurate measures for residents, mobile and migrant populations will help control reemergence of the disease.

Ecological niche models can calculate the potential distribution of the species based on geographical location of species and environmental resources variables (3). The maximum entropy model had been widely used to predict the distribution conditions of species among all ecological niche models. Given that the suitable distribution of $P$. chinensis demonstrated a great possibility for MT-ZVL reemergence, MaxEnt software (Version 3.4.1; Phillips et al. 2020) was used to calculate distribution of suitable environments for the vector. The study area consisted of 6 endemic provinces: Gansu, Shanxi, Shaanxi, Sichuan, Henan, and Hebei. These provinces are located between latitude $26.05^{\circ}-42.79^{\circ} \mathrm{N}$ and longitude $92.34^{\circ}-$ $119.85^{\circ} \mathrm{E}$. A completed survey on historical literatures was conducted, which were published on CNKI (https://www.cnki.net/) and Wanfang Data (http:// www.wanfangdata.com.cn/index.html) between 1950 and 2017, and the distribution points of $P$. chinensis were collected. The national field survey data of $P$. chinensis in 2016 was collected from national repository. The environmental variables included 24 datasets of bioclimatic and geographical data (Table 1), which was generally recognized as important factors for sandfly survival (3-4). ArcGIS10.7 was used to construct and process the environmental variable datasets, and the distribution datasets of $P$. chinensis were then imported into MaxEnt software as the biological training data. Overall, $75 \%$ of the data served as the training dataset, and the remaining $25 \%$ of the sample points were randomly selected as test data. The results of the model displayed the potential ecological suitability of the vector to survive as a range from 0 (unsuitable) to 1 (suitable), and we defined 
TABLE 1. Environmental variables used in MaxEnt model.

\begin{tabular}{|c|c|c|c|c|c|}
\hline $\begin{array}{c}\text { Variable } \\
\text { Classification }\end{array}$ & Variable Name & Definition & Units & Variable Type & Source \\
\hline \multicolumn{6}{|l|}{ Bioclimatic Data } \\
\hline & BIO_01 & Annual mean temperature & ${ }^{\circ} \mathrm{C}$ & \multirow{19}{*}{ continuous } & \multirow{19}{*}{$\begin{array}{l}\text { https://www.worldclim. } \\
\text { org/data/worldclim21. } \\
\text { html }\end{array}$} \\
\hline & BIO_02 & Mean diurnal range & ${ }^{\circ} \mathrm{C}$ & & \\
\hline & BIO_03 & Isothermality & - & & \\
\hline & BIO_04 & Standard deviation of temperature seasonality & - & & \\
\hline & BIO_05 & Max temperature of warmest month & ${ }^{\circ} \mathrm{C}$ & & \\
\hline & BIO_06 & Min temperature of coldest month & ${ }^{\circ} \mathrm{C}$ & & \\
\hline & BIO_07 & Temperature annual range & ${ }^{\circ} \mathrm{C}$ & & \\
\hline & BIO_08 & Mean temperature of wettest quarter & ${ }^{\circ} \mathrm{C}$ & & \\
\hline & BIO_09 & Mean temperature of driest quarter & ${ }^{\circ} \mathrm{C}$ & & \\
\hline & BIO_10 & Mean temperature of warmest quarter & ${ }^{\circ} \mathrm{C}$ & & \\
\hline & BIO_11 & Mean temperature of coldest quarter & ${ }^{\circ} \mathrm{C}$ & & \\
\hline & BIO_12 & Annual precipitation & $\mathrm{mm}$ & & \\
\hline & BIO_13 & Precipitation of wettest month & $\mathrm{mm}$ & & \\
\hline & BIO_14 & Precipitation of driest month & $\mathrm{mm}$ & & \\
\hline & BIO_15 & Coefficient of variation of precipitation seasonality & - & & \\
\hline & BIO_16 & Precipitation of wettest quarter & $\mathrm{mm}$ & & \\
\hline & BIO_17 & Precipitation of driest quarter & $\mathrm{mm}$ & & \\
\hline & BIO_18 & Precipitation of warmest quarter & $\mathrm{mm}$ & & \\
\hline & BIO_19 & Precipitation of coldest quarter & $\mathrm{mm}$ & & \\
\hline \multicolumn{6}{|l|}{ Geographical Data } \\
\hline & LU & Land use & - & categorical & \multirow{4}{*}{ http://www.resdc.cn/ } \\
\hline & LF & Landform & - & categorical & \\
\hline & NDVI & Annual normalized difference vegetation index & - & continuous & \\
\hline & VEG & Vegetation & - & categorical & \\
\hline & ELV & Elevation & $\mathrm{m}$ & continuous & $\begin{array}{l}\text { https://www.worldclim. } \\
\text { org/data/worldclim21. } \\
\text { html }\end{array}$ \\
\hline
\end{tabular}

areas with ecological suitability values of $0-0.3$, $0.3-0.6$, and $0.6-0.98$ as being at low, medium, and high-risk survival area, respectively.

The average area under the curves (AUC) was 0.936 (with a standard deviation of 0.011 ), which indicated excellent prediction accuracy and effect. The results of the jackknife test of variable importance revealed that annual normalized difference vegetation index (NDVI) was the most important factor and contributed the most to the model when the other variables remained stable. Other important individual factors were land use (LU), annual mean temperature (BIO_01), vegetation (VEG), landform (LF), and mean temperature of coldest quarter (BIO_11) (Figure 1A). Response curves showed the ecological suitability of $P$. chinensis was relatively high when the NDVI was $\geq$
0.2 ; when the land consisted of forests, rural agricultural areas, and mining areas; when the average annual temperature was $\geq 10{ }^{\circ} \mathrm{C}$; when the vegetation was shrubs or meadow; and when the mean temperature during the cold and dry quarter was between $-5^{\circ} \mathrm{C}$ and $-10{ }^{\circ} \mathrm{C}$ (Figure $1 \mathrm{~B}$ ).

According to the result, the suitable distribution of $P$. chinensis presented wide spatial distribution and high indigenous spatial clustered characteristics, which indicated high survival probability. They would survive in the following suitable high-risk areas: the temperate hills of northern Sichuan and southern Gansu, the Loess Plateau region (eastern and central Gansu, Guanzhong area in Shaanxi, and most areas of Shanxi and northwestern Henan) and southern Hebei (Figure 1C). 
A

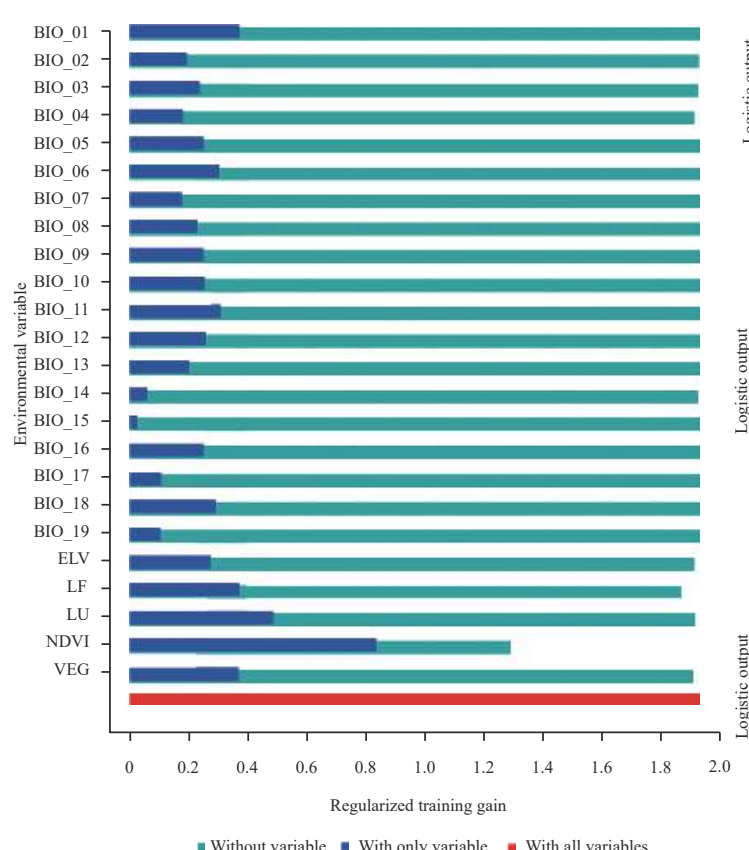

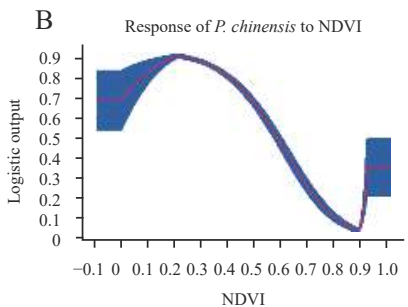

NDVI

Response of $P$. chinensis to BIO 01

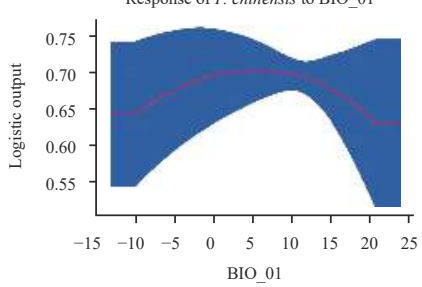

Response of P. chinensis to LF

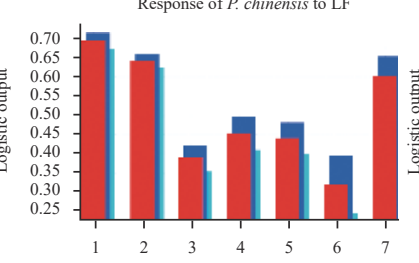

LF

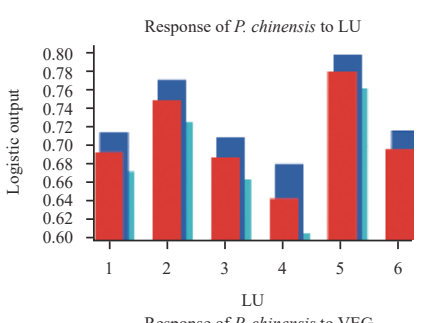

Response of $P$. chinensis to VEG

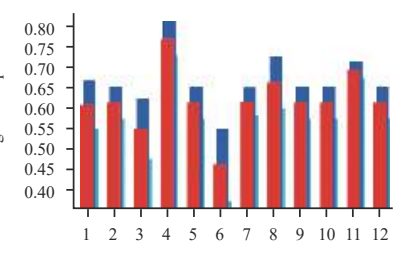

VEG

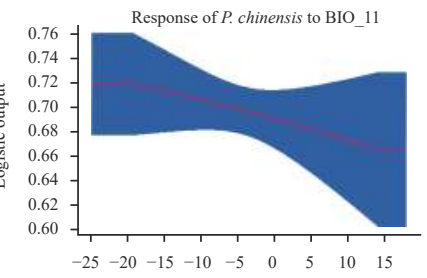

BIO_11

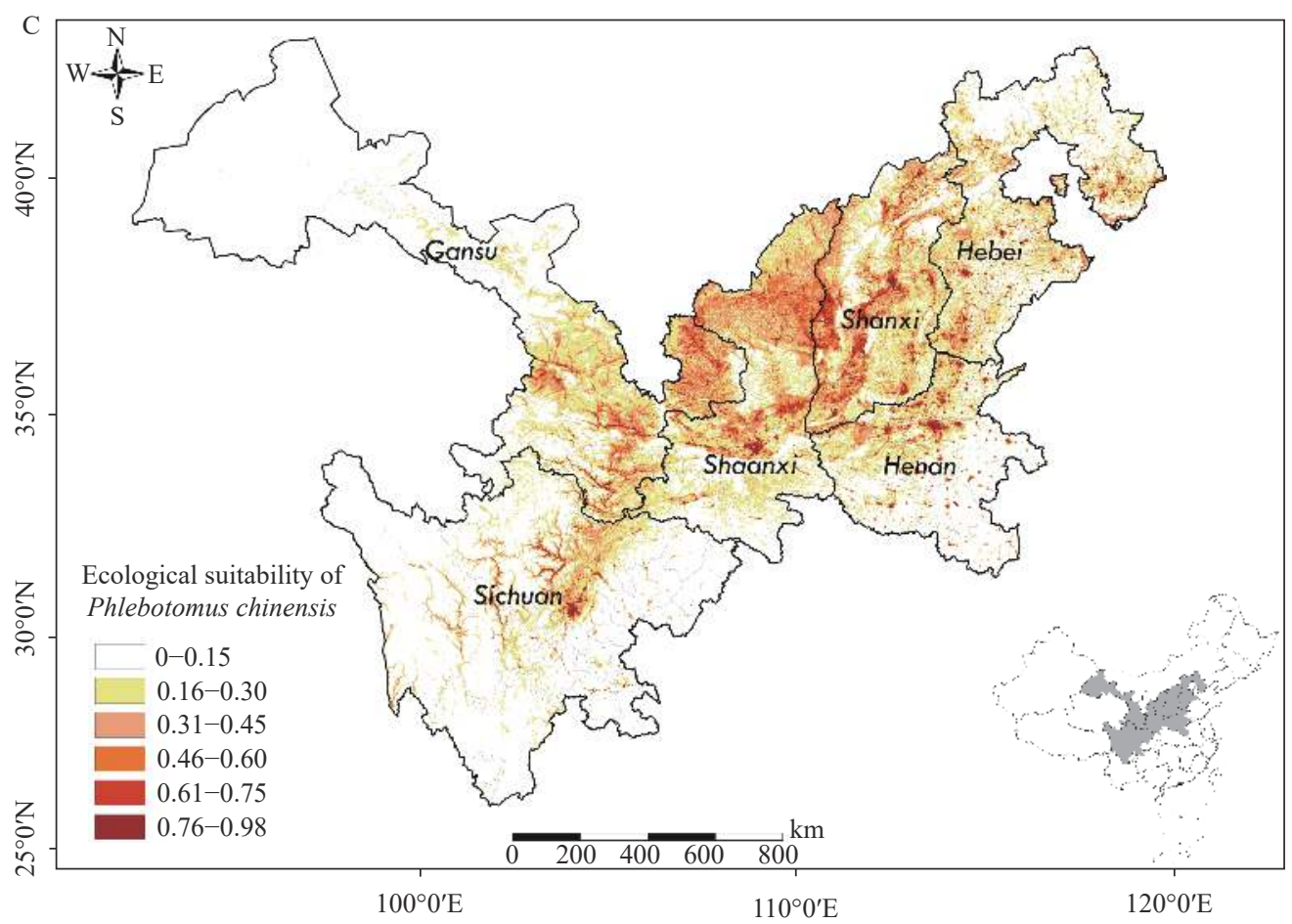

FIGURE 1. Results of the MaxEnt model: Analysis of environmental variable contributions and point-wise mean ecological suitability of suitable distribution for Phlebotomus chinensis ( $P$. chinensis). (based on $P$. chinensis distribution data documented in literatures between 1950-2017). (A) Jackknife test results of regularized training gains for $P$. chinensis; (B) Response curves of 6 major environmental variables (NDVI, LU, BIO_01, VEG, LF, and BIO_11); (C) The distribution of suitable environments for $P$. chinensis in MT-ZVL endemic provinces in China. Abbreviations: NDVI=Annual normalized difference vegetation index, LU=Land use, BIO_01=Annual mean temperature, VEG=Vegetation, LF=Landform, BIO_11=Mean temperature of coldest quarter. In Figure 1A, blue and red bars showed training gain of variables if the model was run in isolation and of all variables, respectively. In Figure 1B, the response curves showed how each environmental variable affected the MaxEnt prediction when all other variables kept average value plotted by the value of each variable on the horizontal axis and training gain on the vertical. 


\section{DISCUSSION}

MT-ZVL and the main vector, $P$. chinensis have been controlled successfully since the 1970s in China. However, the reported numbers of MT-ZVL indigenous cases increased gradually in recent years (1-2). The prediction results showed a wide spatial distribution of suitable environments for $P$. chinensis in China with high indigenous clustered characteristics. The most suitable areas for $P$. chinensis were generally temperate hilly zones with a moderate NDVI value; where the land mainly consisted of forests, suburban agricultural, and industrial land; with an average annual temperature of about $10{ }^{\circ} \mathrm{C}$; where shrubs or meadows were the main vegetation; and where the mean temperature during the cold and dry quarters were $-5{ }^{\circ} \mathrm{C}$ to $-10{ }^{\circ} \mathrm{C}$. This suggested that suitable living conditions for the high-density $P$. chinensis that caused the reemergence of MT-ZVL already existed. The study aimed to identify key environmental factors for $P$. chinensis surviving and to control reemergence of MT-ZVL in China. Key environments should get more attention and monitoring. Furthermore, this study provided more evidence to discover the potential risk of MT-ZVL transmission to concentrate resources for accurate prevention and control.

Research showed that bioclimatic factors such as temperature and precipitation changes caused by climate warming could allow $P$. chinensis to survive at higher latitudes and altitudes (3). Different bioclimatic and geographical factors in various regions have a vital influence on the distribution of sandflies (4). The policies of returning farmland to forests and closing hills for afforestation have increased the density of wild $P$. chinensis, and the natural foci in hilly zones has further spread the transmission risk of MT-ZVL to surrounding areas. Therefore, understanding the influence of bioclimatic and geographical factors on the suitable distribution of the vector can scientifically explain the reemergence of MT-ZVL to a certain extent.

High temperatures will lead to excessive larval metabolic consumption and death, and larvae at higher altitudes will take longer to develop (5). Vegetation and NDVI have important influences on the reproduction and development of wild-type $P$. chinensis. The development of females depends on absorbing the blood of livestock grazing in meadows, whereas that of males mainly depends on soft-stemmed plants (4). Suburban agricultural, industrial, and mining lands have proven suitable as temporary habitats for semi-wild-type $P$. chinensis vectors (G), and this factor has also led to an increased ecological suitability of the vector in some large cities.

Monitoring key environments suitable for $P$. chinensis surviving should be strengthened in southern Gansu and northern Sichuan, and spraying the wildtype $P$. chinensis in key natural caves (the natural foci for MT-ZVL) with residual insecticides is recommended ( 7 ). Combining insecticide spraying measures for the semi-wild-type $P$. chinensis in cavehouses that are abandoned or in use and livestock compounds would be effective in endemic areas in the Loess Plateau region $(6,8)$. Second, accurate prevention and control measures should be taken in high-risk areas. Residents should be reminded to strengthen protective measures to avoid being bitten by $P$. chinensis. Diagnosis, screening, and health education should also be enhanced among mobile and migrant populations, considering frequent population movements increase the risk of MT-ZVL importation in non-endemic areas (9).

However, the present study has some limitations. Most of the historically-endemic counties with reemergent MT-ZVL were predicted as high-risk areas for $P$. chinensis surviving, but the vector's high ecological suitability value in some large cities deviated from the actual distribution of the disease. One possible reason is that the distribution points of the vector in suburban and rural areas has been eliminated during the Patriotic Health Campaign and previous urbanization of rural areas. Moreover, socioeconomic factors like population density and gross domestic product (GDP) were not considered, and canines were not addressed. These factors should be included in the future to more comprehensively assess the distribution of the $P$. chinensis vector.

Acknowledgments: Many thanks to the staff of National Institute of Parasitic Diseases, Chinese Center for Diseases Control and Prevention for their kind help on professional consulting and data providing.

Conflicts of interest: No conflicts of interest were reported.

Fundings: Supported by National Special Science and Technology Project for Major Infectious Diseases of China (No. 2016ZX10004222-004) and Research on prediction and Early warning and Intervention technology of meteorological sensitive parasitic diseases (No. 2017FY101203). Science and technology research project of Shanghai Municipal Health Commission (No. 20194Y0359, 20174Y0188). 
doi: $10.46234 / \mathrm{ccdcw} 2020.223$

\# Corresponding author: Shizhu Li, lisz@chinacdc.cn.

\begin{abstract}
National Institute of Parasitic Diseases, Chinese Center for Disease Control and Prevention; Chinese Center for Tropical Diseases Research;Key Laboratory of Parasite and Vector Biology, Ministry of Health; WHO Collaborating Center for Tropical Diseases;National Center for International Research on Tropical Diseases, Ministry of Science and Technology, Shanghai, China; ${ }^{2}$ School of Global Health, Chinese Center for Tropical Diseases Research, Shanghai Jiao Tong University School of Medicine, Shanghai, China.
\end{abstract}

Submitted: September 29, 2020; Accepted: October 14, 2020

\section{REFERENCES}

1. Zhou ZB, Wang JY, Gao CH, Han S, Li YY, Zhang Y, et al. Chapter five - contributions of the national institute of parasitic diseases to the control of visceral leishmaniasis in China. Adv Parasitol 2020;110:185 216. http://dx.doi.org/10.1016/bs.apar.2020.04.003.

2. Zhou ZB, Lyu S, Zhang Y, Li YY, Li SZ, Zhou XN. Preplanned studies: visceral leishmaniasis - China, 2015-2019. China CDC Wkly 2020;2(33):625 - 8. http://dx.doi.org/10.46234/ccdcw2020.173.

3. Carvalho BM, Rangel EF, Ready PD, Vale MM. Ecological niche modelling predicts southward expansion of Lutzomyia (Nyssomyia) Flaviscutellata (Diptera: Psychodidae: Phlebotominae), vector of
Leishmania (Leishmania) amazonensis in south America, under climate change. PLoS One 2015;10(11):e0143282. http://dx.doi.org/10.1371/ journal.pone.0143282.

4. Gao X, Xiao JH, Liu BY, Wang HB. Impact of meteorological and geographical factors on the distribution of Phlebotomus chinensis in northwestern mainland China. Med Vet Entomol 2018;32(3):365 - 71 http://dx.doi.org/10.1111/mve.12307.

5. Xiong GH, Jin CF, Chen XZ, Hong YM, Su ZW, Liu PZ, et al. Studies on the binomics of sandfly, phlebotomus chinensis newstead, 1916, and relation to the visceral leishmaniasis in southern Gansu and northern Sichuan, China. Wuyi Sci J 1992(1):7 - 18, 406. http://dx.doi.org/ 10.15914/j.cnki.wykx.1992.00.001. (In Chinese).

6. Xiong GH, Jin CF. Research progress on the resting habits of major sandflies and control strategy. Chin J Parasitol Parasit Dis 2006;24 (4):293 - 8. http://dx.doi.org/10.3969/j.issn.1000-7423.2006.04.013. (In Chinese).

7. Li Y, Li C, Yang ZX, Wu Y, Yuan YF, Chen YM, et al. New trends and countermeasures of visceral leishmaniasis endemic in Wudu District, Longnan City, Gansu Province. Endem Dis Bull 2010;25(2):38 - 41. http://dx.doi.org/10.13215/j.cnki.jbyfkztb.2010.02.033. (In Chinese).

8. Chen HM, Chen HY, Gao JP, Li KL, Yang ZZ, Peng H, et al. Ecological niches of sandfly (Diptera: Psychodidae) in the extension region of Loess Plateau, China: an endemic focus of visceral leishmaniasis. Chin J Vector Biol Control 2019;30(6):597 - 602. http://dx.doi.org/10.11853/j.issn. 1003.8280.2019.06.001. (In Chinese).

9. Zhou ZB, Li YY, Zhang Y, Li SZ. Prevalence of visceral leishmaniasis in China in 2019. Chin J Parasitol Parasit Dis 2020;38(5):1-6. http://dx.doi.org/10.12140/j.issn.1000-7423.2020.05.001. (In Chinese). 